\title{
Slow light with low dispersion and nonlinear enhancement in a lattice-shifted photonic crystal waveguide
}

\author{
Yohei Hamachi, ${ }^{1,2}$ Shousaku Kubo, ${ }^{1,2}$ and Toshihiko Baba ${ }^{1,2, *}$ \\ ${ }^{1}$ Department of Electrical and Computer Engineering, Yokohama National University, 79-5 Tokiwadai, Hodogaya-ku, \\ Yokohama 240-8501, Japan \\ ${ }^{2}$ CREST, Japan Science and Technology Agency, 5 Sanbancho, Chiyoda-ku Tokyo 102-0075, Japan \\ *Corresponding author: baba@ynu.ac.jp
}

Received November 13, 2008; revised January 14, 2009; accepted February 23, 2009; posted March 5, 2009 (Doc. ID 104109); published March 26, 2009

\begin{abstract}
We discovered that a silicon-on-insulator photonic crystal waveguide whose lattice is shifted along the waveguide generates wideband, low-dispersion, slow light with excellent reproducibility. We observed delayed transmission of picosecond optical pulses, as well as two-photon absorption and self-phase modulation enhanced by a high internal light intensity in the slow-light regime. () 2009 Optical Society of America OCIS codes: $230.5298,230.7370$.
\end{abstract}

Slow light having a small group velocity $v_{\mathrm{g}}$ is expected to be useful in achieving optical buffers and nonlinear enhancements. Photonic nanostructures have been studied to generate on-chip slow light at room temperature [1,2]. In this Lettter, we report slow light generated in a photonic crystal waveguide (PCW). Slow light in standard PCWs, consisting of a line defect in a photonic crystal slab, occurs near the photonic band edge. It is usually accompanied by a large group-velocity dispersion (GVD), which severely deforms optical pulses and disturbs these applications. This problem can be eliminated by internal compensation of the GVD [3-5] and/or by suppression of the GVD by band engineering [6]. In particular, the latter approach maintains the pulse shape inside the device, so that the optical pulse is compressed in space due to the low $v_{\mathrm{g}}$, and the peak intensity of the pulse increases and the interaction time is prolonged, resulting in an enhancement of the nonlinearities to scale with the square of the group index. We call this low-group-velocity and lowdispersion (LVLD) slow light. Such LVLD has been observed in PCWs, when the diameter of the airholes in the photonic crystal slab adjacent to the line defect is reduced while simultaneously other airholes are enlarged $[7,8]$. Alternatively, the positions of the first and second rows of airholes away from the line defect are shifted in the direction normal to the line defect [9]. However, this requires precise control of multiple parameters whose values must be simultaneously adjusted if one desires LVLD in a target wavelength range. Furthermore, the group velocity $v_{\mathrm{g}}$ and bandwidth $\Delta \lambda$ cannot be changed independently of the wavelength range. In this study, we discovered that a shift of the third row of airholes in the direction parallel to the line defect permits the observation of LVLD in a target wavelength range with a simpler structural optimization and higher reproducibility. We fabricated a LVLD-PCW and observed a slowlight pulse showing enhanced nonlinearities.

Figure 1 shows a PCW consisting of a single line defect and triangular lattice airholes, formed in an $\sim 210$-nm-thick Si air-bridge membrane on a siliconon-insulator substrate by using electron-beam lithography and $\mathrm{SF}_{6}$ inductively coupled plasma etching. The lattice constant $a$ was $450 \mathrm{~nm}$, and the airhole diameter $2 r$ was $284 \mathrm{~nm}$. The third row of airholes was shifted by a distance $s$. Except for adiabatic regions near the input and output ends, which improve the light coupling into the slow-light mode, the central region used for generating the LVLD was $390 \mu \mathrm{m}$ long.

Figure 2(a) shows the calculated photonic bands for transverse-electric polarization, where the twodimensional approximation with an equivalent index of 2.9 is used. The normalized airhole diameter $2 r / a$ is fixed at 0.75 . As $s$ increases, the slab mode band on the low-frequency side pushes the guided mode up and deforms its band shape. For $s / a=0.10$, the band is nearly linear between the indicated two open circles. Higher-order dispersion components including the GVD are then completely eliminated. For larger values of $s / a$, the band doubly bends, exhibiting LVLD around the central inflection point. In general, the band slope increases, the group index $n_{\mathrm{g}}$ $\equiv c / v_{\mathrm{g}}$ (where $c$ is the speed of light in vacuum) decreases, and $\Delta \lambda$ is broadened as $s$ increases. An im-

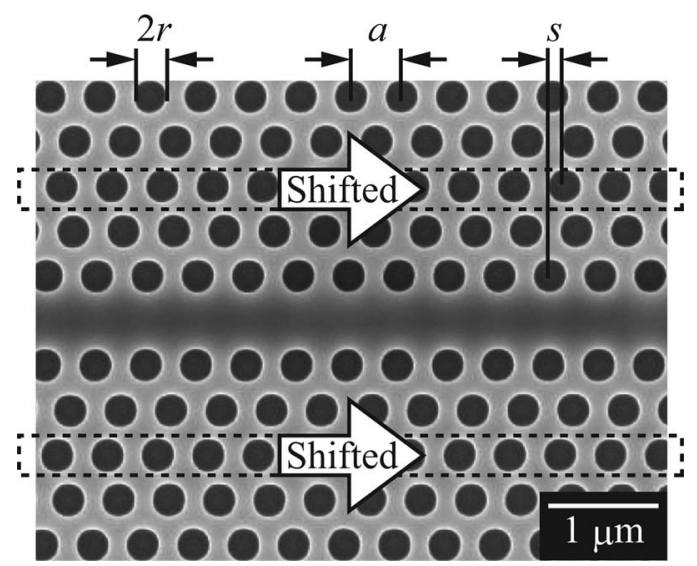

Fig. 1. Top view of fabricated LVLD-PCW. 

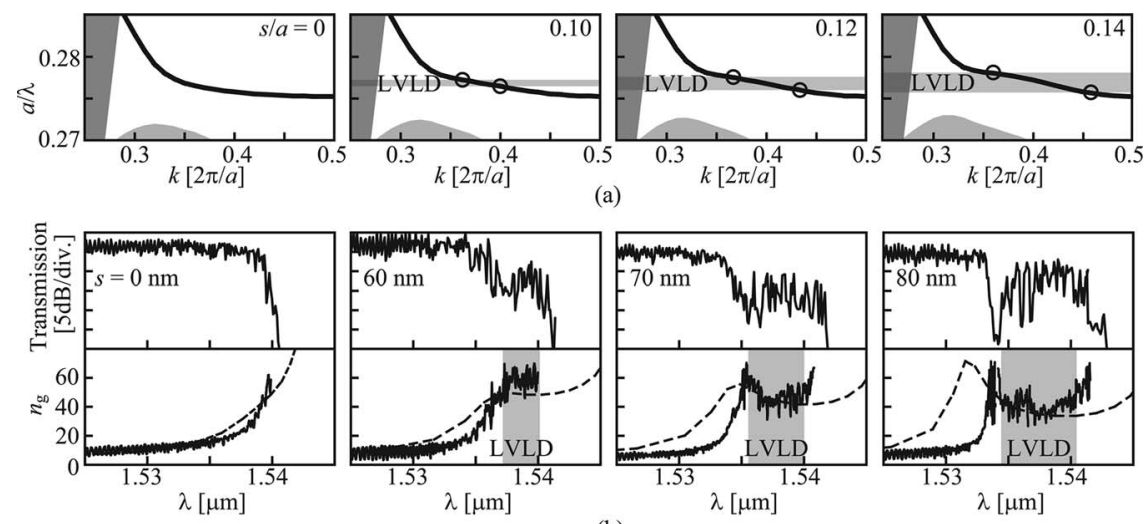

Fig. 2. (a) Calculated photonic bands and (b) measured transmission and group index spectra for different shifts $s$. In (a), the dark areas at the left and bottom denote the air light cone and the slab mode region, respectively. In (b), the dotted curves are the calculated spectra.

portant advantage of this LVLD is that it is easy to observe experimentally, because $s$ is the only optimization parameter and the wavelength range is not sensitive to $s$ but only to $2 r / a$.

Figure 2(b) shows the transmission and group index spectra measured by the modulation phase shift method [4]. The dotted curves are the calculated group index spectra. The experimental plots show LVLD in good agreement with these calculated results. The oscillations in the spectra are due to an internal Fabry-Perot resonance. Neglecting this oscillation and defining the LVLD range $\Delta \lambda$ to be the value that gives a $\pm 10 \%$ variation in $n_{\mathrm{g}}$, we obtain $n_{\mathrm{g}}=60$ and $\Delta \lambda=3.1 \mathrm{~nm}$ for $s=60 \mathrm{~nm}, n_{\mathrm{g}}=47$ and $\Delta \lambda$ $=4.5 \mathrm{~nm}$ for $s=70 \mathrm{~nm}$, and $n_{\mathrm{g}}=40$ and $\Delta \lambda=6 \mathrm{~nm}$ for $s=80 \mathrm{~nm}$. The maximum value of the normalized delay-bandwidth product $n_{\mathrm{g}}(\Delta \omega / \omega)$ was 0.16 at $s / a$ $=0.14$. It is nearly half of that for another type of LVLD waveguide [9]. It is due to the band profile of our waveguide, in which the straight part appears separately from the horizontal part and so the range of the straight part is slightly narrower than that in [9]. This is a disadvantage of our waveguide, while the simple fabrication and reproducibility of the LVLD characteristics are big advantages. As LVLD is observed repeatedly in many samples, its reproducibility is confirmed. A large transmission dip is also observed repeatedly between the fast-light regime on the short wavelength side and the slow-light regime of the LVLD. The group index could not be measured at the dip because of the low intensity. It might be due to a rapid change in the modal field with wavelength, which particularly increases the propagation loss and/or coupling loss at the ends of the waveguide.

The LVLD enables the propagation of slow-light pulses. We inserted optical pulses of $0.6 \mathrm{ps}$ FWHM to the PCW. Figure 3 shows the delay spectrum of another sample and cross-correlation traces of the output pulse with a reference pulse of the same FWHM. In the fast-light regime $\left(n_{\mathrm{g}} \sim 8\right)$, the delay $\Delta t$ and the FWHM $\Delta \tau$ of the output pulse are 10 and $1.9 \mathrm{ps,}$ respectively. In the slow-light regime $\left(n_{\mathrm{g}} \sim 30\right)$, on the other hand, they are 40 and $2.4 \mathrm{ps}$, respectively. Dispersion in the slow-light regime is suffi- ciently small that it can be observed in the output pulse.

Next, we evaluated the nonlinearity of the PCW. Figure 4 compares the intensity responses for two different samples exhibiting fast $\left(n_{\mathrm{g}} \sim 8\right)$ and slow $\left(n_{\mathrm{g}} \sim 30\right)$ light at the same wavelength range around $1.554 \mu \mathrm{m}$. Here, the FWHM of the initial pulse spectrum is narrowed using a bandpass filter so that $\Delta \tau$ broadens to $4.1 \mathrm{ps}$. The peak power of the input pulse $P$ launched inside the PCW is estimated from the time-averaged power, repetition frequency, $\Delta \tau$ at the input end, and total loss between the laser source and the PCW. An almost linear response is observed in the fast-light regime. On the other hand, saturation is observed for $P>0.3 \mathrm{~W}$ for the slow-light regime. It probably arises from two-photon absorption (TPA) and accompanied free carrier absorption in the Si layer. Similar characteristics have been reported for Si photonic wire waveguides (PWWs) [10,11]. However, in those previous studies, the input power level was of the order of $1-10 \mathrm{~W}$, and the device length was of the order of $1 \mathrm{~mm}$. In contrast, we observe the saturation for lower power and shorter device lengths, even though the mode cross-sectional area is slightly larger, and the carrier lifetime is as short as $<100 \mathrm{ps}$, which is caused by carrier diffusion in the photonic crystal slab and greatly reduces the free carrier absorption. Using Eq. (1) in [10], we calculated the intensity response, as shown by the solid curves in Fig. 4. Here, we assumed a carrier lifetime

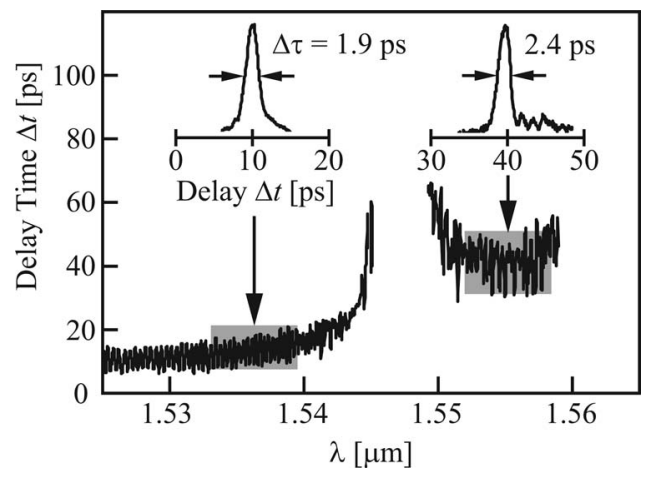

Fig. 3. Delay spectrum and cross-correlation traces of the output optical pulse in the fast- and slow-light regimes. 


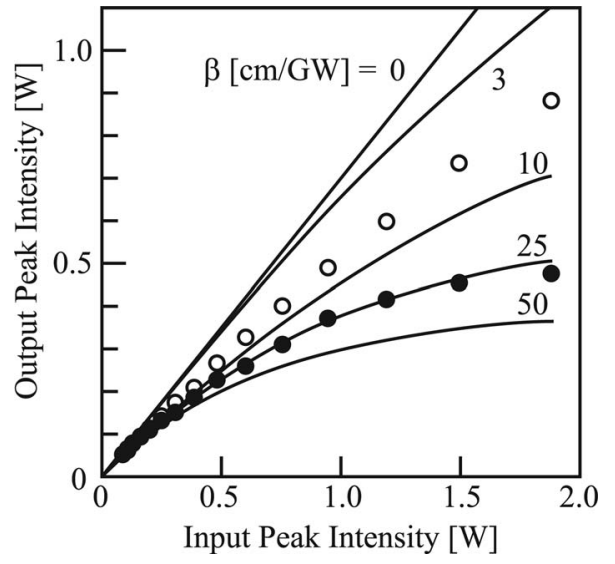

Fig. 4. Intensity response. The solid curves are the calculation results for different effective TPA coefficients $\beta$. The open and closed circles denote the experimental results for two PCWs exhibiting $n_{\mathrm{g}} \sim 8$ and ng $\sim 30$, respectively.

of $70 \mathrm{ps}$ [12], calculated cross-sectional area of $0.4 \mu \mathrm{m}^{2}$ and evaluated propagation loss of 30 and $60 \mathrm{~dB} / \mathrm{cm}$ for the $n_{\mathrm{g}} \sim 8$ and $n_{\mathrm{g}} \sim 30$ waveguides, respectively. The effective TPA coefficient $\beta$ enhanced by the slow-light effect is estimated to be $25 \mathrm{~cm} / \mathrm{GW}$. This value is 42 -fold higher than a reported value in [10] for the Si PWW. This enhancement can be explained by the 6.7-fold larger $n_{\mathrm{g}}$ in the PCW than a standard value of 4.5 in the $\mathrm{Si}$ PWW; the square of the $n_{\mathrm{g}}$ ratio almost corresponds to the enhancement factor of $\beta$. In the same way, $\beta$ is expected to be $3 \mathrm{~cm} / \mathrm{GW}$ for $n_{\mathrm{g}} \sim 8$. But its output intensity is slightly lower from the low- to high-intensity regimes. A reason considered for this is a slightly lower detection efficiency of light output for this sample.

The pulse spectra are also plotted in Fig. 5. The spectra in the absence of a sample exhibit slight broadening caused by self-phase modulation. A small change in the spectral shape is observed in the presence of a sample in the fast-light regime. However, in the slow-light regime, the asymmetry of spectral broadening and a small blueshift are observed for $P$ $>0.3 \mathrm{~W}$, corresponding to the onset of TPA. Therefore, these must be due to the carrier plasma effect accompanied by TPA $[13,14]$.

In summary, we have developed a lattice-shifted $\mathrm{Si}$ photonic crystal waveguide showing LVLD characteristics with excellent reproducibility. We experimentally demonstrated the LVLD in a silicon-oninsulator-based device using a modulation phase shift measurement and short optical pulse transmission. We observed a group index $n_{\mathrm{g}}$ of 25-60 in the wavelength range of $3-12 \mathrm{~nm}$. We observed about 42-fold TPA and accompanying self-phase modulation and free carrier shifts compared with the Si photonic wire waveguides. To our knowledge, this is the first

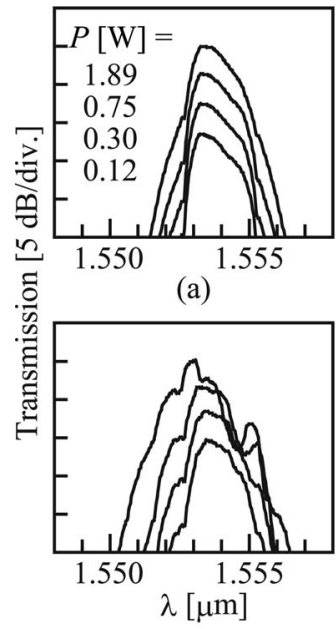

(c)
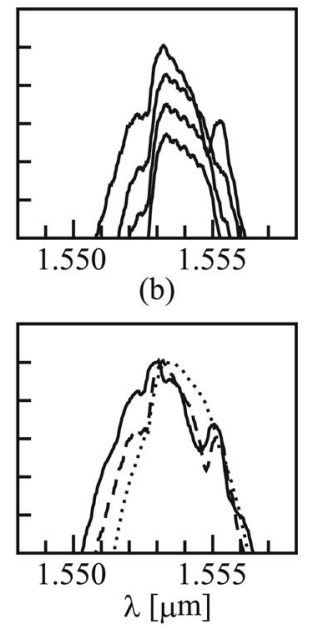

(d)
Fig. 5. Spectra of the input and output optical pulse for different input optical powers $P$. (a) Input spectra. (b) Output spectra for $n_{\mathrm{g}} \sim 8$ and (c) $n_{\mathrm{g}} \sim 30$. (d) Comparison between the input spectrum (dotted curve) and output spectra for $n_{\mathrm{g}} \sim 8$ (dashed curve) and $n_{\mathrm{g}} \sim 30$ (solid curve) at $P$ $=1.89 \mathrm{~W}$.

demonstration of a nonlinear enhancement by slow light in a PCW.

This work was partly supported by a Grant-in Aid from the Priority Area of the Ministry of Education, Culture, Sports, Science and Technology (MEXT).

\section{References}

1. T. Baba, Nat. Photonics 2, 465 (2008).

2. T. F. Krauss, J. Phys. D 40, 2666 (2007).

3. D. Mori and T. Baba, Appl. Phys. Lett. 85, 1101 (2004).

4. T. Baba, T. Kawasaki, H. Sasaki, J. Adachi, and D. Mori, Opt. Express 16, 9245 (2008).

5. M. L. Povinelli, S. G. Johnson, and J. D. Joannopoulos, Opt. Express 13, 7145 (2005).

6. A. Y. Petrov and M. Eich, Appl. Phys. Lett. 85, 4866 (2004).

7. L. H. Frandsen, A. V. Lavrinenko, J. F. Pedersen, and P. I. Borel, Opt. Express 14, 9444 (2006).

8. S. Kubo, D. Mori, and T. Baba, Opt. Lett. 32, 2981 (2007)

9. J. Li, T. P. White, L. O'Faolain, A. G. Iglesias, and T. F. Krauss, Opt. Express 16, 6227 (2007).

10. H. Yamada, M. Shirane, T. Chu, H. Yokoyama, S. Ishida, and Y. Arakawa, Jpn. J. Appl. Phys. 44, 6541 (2005).

11. E. Dulkeith, Y. A. Vlasov, X. Chen, N. C. Panoiu, and R. M. Osgood, Opt. Express 14, 5524 (2006).

12. T. Tanabe, M. Notomi, S. Mitsugi, A. Shinya, and E. Kuramochi, Opt. Lett. 30, 2575 (2005).

13. H. Oda, K. Inoue, Y. Tanaka, N. Ikeda, Y. Sugimoto, H. Ishikawa, and K. Asakawa, Appl. Phys. Lett. 90, 231102 (2007).

14. L. Yin and G. P. Agrawal, Opt. Lett. 32, 2031 (2007). 